\title{
Cerebellar Liponeurocytoma with High Proliferation Index: Treatment Options
}

\author{
C.E. Châtillon, M.C. Guiot, D. Roberge, R. Leblanc
}

Can. J. Neurol. Sci. 2009; 36: 658-661

First described by Bechtel and collaborators ${ }^{1}$, cerebellar liponeurocytomas are posterior fossa lesions composed of densely packed neuronal cells admixed with foci of welldifferentiated adipocyte-like cells. Similar tumors have also been referred to as lipomatous medulloblastoma, lipidized medulloblastoma, medullocytoma, neurolipocytoma, lipomatous glioneurocytoma, or lipidized mature neuroectodermal tumor of the cerebellum ${ }^{2-8}$. These different appellations reflect the histopathological similarities between liponeurocytomas, medulloblastomas and central neurocytomas. However, cerebellar liponeurocytoma is genetically distinct from medulloblastoma and central neurocytoma ${ }^{9}$; it is now recognized as a separate entity by the World Health Organization ${ }^{10}$. Because of the small number of reported cases, the proper management of cerebellar liponeurocytoma, especially when subtotally resected, remains to be elucidated. We report the case of a patient with a hemispheric cerebellar liponeurocytoma in which the proliferation index contributed to the decision to use adjuvant radiotherapy after gross total resection.

\section{CASE REPORT}

A 42-year-old, right-handed woman gave a six-month history of nuchal pain, paraesthesias of the right hemi-tongue, dysarthria, mild ataxia of the right upper extremity and unsteadiness of gait. General physical examination was unremarkable. Neurological examination revealed dysmetria of the right upper extremity and decreased rapid alternating movements of the right hand and foot. Her gait was unsteady and she had a diminished ability to tandem walk. Computed tomography (CT) scanning revealed a well-demarcated, irregularly enhancing, heterogenous mass within the right cerebellar hemisphere, measuring five centimeters in largest diameter, and exhibiting areas of fat density (Figure 1A, B). Magnetic resonance imaging (Figure 1C) showed spontaneously hyperintense areas on T1-weighted sequences correlating with the CT hypodense areas, confirming the presence of fat within the tumor.

The patient underwent a gross total resection of the tumor through a right suboccipital approach. Microscopic examination revealed a monotonous cellular proliferation with focal areas of lipomatous differentiation (Figure 2A, B). The cells had mostly
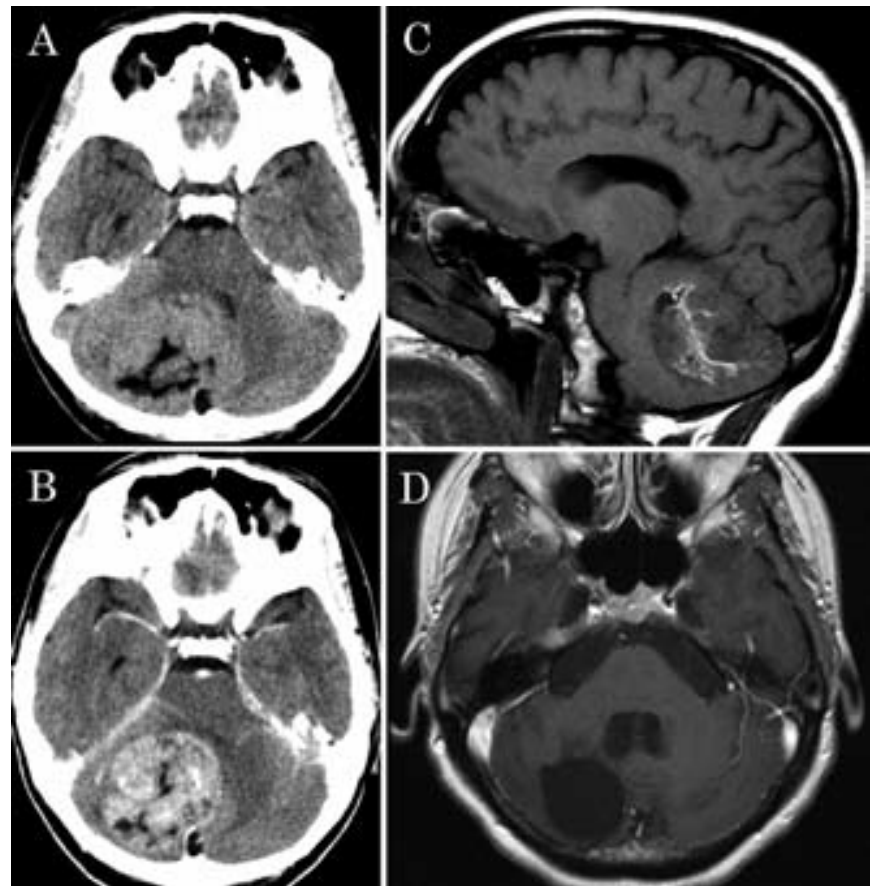

Figure 1: Radiological appearance of the lesion. (A) Axial plain CT showing a large $(5 \times 4.5 \mathrm{~cm})$ posterior fossa lesion, involving mostly the right cerebellar hemisphere. There is significant local mass effect with near-obliteration of the 4th ventricle and compression of the ipsilateral brachium pontis. The lesion is heterogeneous with isodense and hypodense (to cortex) components. The CT images were inconclusive but suggestive of an extra-axial lesion arising from the tentorium cerebelli. (B) Axial CT with contrast elicits heterogeneous enhancement of the tumor. (C) Sagittal T1 plain confirms the presence of fat within the tumor, as areas of spontaneous hyperintensity correspond to the hypodense areas on CT. (D) Axial global with gado at three year follow-up reveals the surgical cavity without evidence of recurrence.

rounded to oval nuclei with open chromatin. However, in some areas the nuclei appeared more elongated and hyperchromatic. Perivascular hyaline deposits and pseudorosettes were noted. A few foci of endothelial proliferation and one small area of necrosis were present. Two mitotic figures per high-power field

From the Department of Neurosurgery (CEC, RL), Department of Neuropathology (MCG), Division of Radiation Oncology (DR), McGill University Health Centre, Montreal, Quebec, Canada.

Received December 9, 2008. Final Revisions Submitted April 23, 2009.

Correspondence to: C.E. Châtillon, Department of Neurosurgery, Montreal Neurological Hospital, McGill University Health Centre, Montreal, Quebec, Canada; 


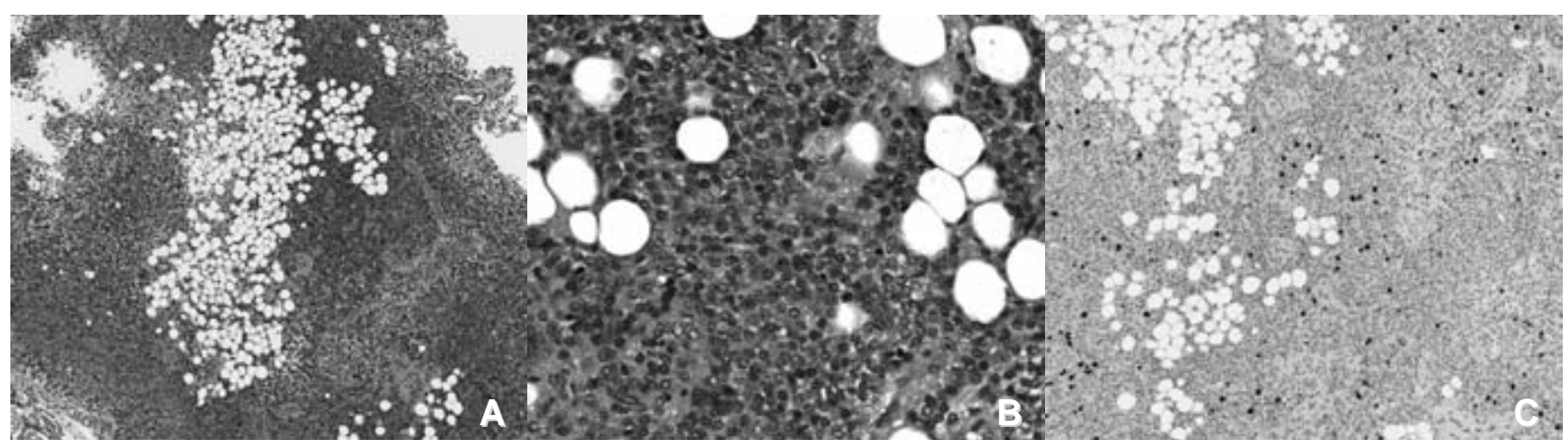

Figure 2: Microscopic images of the lesion. (A) Hematoxylin and eosin $(H \& E)$ stain at low magnification (4x) showing the monotonous neuronal proliferation and an area of adipocytic differentiation. (B) Higher magnification (20x) magnification $H$ \& E showing the highly cellular homogeneous population of neuronal cells with ovoid nuclei and loose chromatin, admixed with adipocyte-like cells. (C) MIB-1 (Ki67) immunostaining (magnification $4 x$ ) within a focus of higher proliferation (10\%).

were seen. Focal areas of lipomatous differentiation were distributed throughout the tumor. Immunohistochemistry was positive for synaptophysin and MAP-2, and sparsely positive for GFAP. The proliferation index (MIB-1) was evaluated at $5 \%$ in most areas but several foci reached $10 \%$ (Figure 2C). Electron microscopy showed the presence of dense core granules and rare synapse-like structures. Based on these findings, the diagnosis of cerebellar liponeurocytoma was made.

Recovery was complicated by a left lower extremity deep vein thrombosis that was treated with six months of anticoagulation. The patient remained with mild, but slightly improved, right upper extremity dysmetria. The tongue paraesthesias and unsteady gait had resolved at the one year follow-up visit.

The option of adjuvant radiotherapy was offered and accepted by the patient. Using 3D conformal radiotherapy, a total dose of 54 Gy was delivered in 30 daily fractions to a clinical target volume that included the surgical cavity and 1 centimetre of surrounding tissue. The treatment was delivered uneventfully with transient focal alopecia, asthenia and dysgeusia. Small areas of T2-hyperintensity in the occipital white matter appeared on the one-year follow-up MRI. These were initially thought to represent radiotherapy-induced changes, but are now felt to represent an unassociated demyelinating disease. The patient remains well and without radiological evidence of recurrence three years after the resection (Figure 1D).

\section{DISCUSSION}

Cerebellar liponeurocytoma is a cerebellar tumor that usually presents in adulthood. It is characterized by densely packed neuronal cells with rare Homer-Wright rosettes and pseudorosettes admixed with foci of well-differentiated adipocyte-like cells $\mathrm{s}^{2,8,9,11,12}$. Areas of neuronal proliferation are

Table 1: Outcome based on initial surgical and adjuvant treatments

\begin{tabular}{lcccc}
\hline Initial treatment & Number of cases & $\begin{array}{c}\text { Recurrence } / \\
\text { progression }\end{array}$ & Death & $\begin{array}{c}\text { Median follow-up } \\
\text { (years) }\end{array}$ \\
\hline GTR+RT & 7 & 0 & 1 & 2 \\
GTR only & 10 & 5 & 1 & 6 \\
STR+RT & 3 & 2 & 1.67 \\
\hline
\end{tabular}

GTR: gross total resection, STR: subtotal resection, RT: radiotherapy. No patients underwent subtotal resection without adjuvant radiotherapy. Patients with insufficient data regarding extent of resection were not included $(9,20)$. Note that although recurrence rate in notably lower in the GTR+RT group, median follow-up does not allow for adequate comparison with the GTR only group. 
Table 2: Proliferation index and outcome

\begin{tabular}{lcccc}
\hline MIB-1 & Number of cases & $\begin{array}{c}\text { Recurrence / } \\
\text { progression }\end{array}$ & $\begin{array}{c}\text { Median time to } \\
\text { recurrence (years) }\end{array}$ & $\begin{array}{c}\text { Median follow-up } \\
\text { (years) }\end{array}$ \\
\hline$<6$ & 18 & 5 & 10 & 2.25 \\
$>6$ & 3 & 1 & 3.5 & 3 \\
\hline
\end{tabular}

Tumors with low mitotic rate recurred in $33.3 \%$ of patients, at a median 2.75 years from initial treatment. One of three cases with high proliferation index recurred at 3.5 years.

usually positive for neuronal immunochemistry markers, including MAP-2 and synaptophysin ${ }^{9,10,13}$. Sparse positivity for GFAP in all regions of the tumor has been described ${ }^{9,10}$. Electron microscopy typically reveals cellular structures of neuronal differentiation such as synapses and dense core granules ${ }^{5}$. The proliferation index of this tumor is usually low as measured by Ki-67 (MIB-1), although cases of elevated mitotic activity have previously been reported ${ }^{11,12,14}$. Based on 16 reported cases in 2000 , Kleihues et $\mathrm{a}^{13}$ proposed a WHO grade I or II for this tumor. This grading was maintained in the 2002 WHO classification of central nervous system (CNS) tumors update ${ }^{10}$.

A review of previously reported cases was performed with emphasis on extent of resection, significance of the proliferation index on recurrence and the role of radiotherapy. Twenty cases with documented extent of surgery or radiotherapy were found ${ }^{1-}$ 9,11,12,14-21. Five cases of supratentorial intraventricular liponeurocytoma have also been reported $^{22}$, but will not be discussed here.

\section{Extent of resection}

The rate of recurrence following gross total resection without radiotherapy was $50 \%$ (five of ten cases), but was usually late (median time to recurrence: ten years) $)^{1,4-8,11,12,15,17}$. Within this group, all cases with a documented follow-up of eight years or more had recurred ${ }^{5,6,8}$. The single case that recurred early, at 3.5 years, had a proliferation index of $15 \%$, suggesting a more aggressive tumor ${ }^{11,12,21}$. There were two deaths in the group having undergone gross total resection without radiotherapy ${ }^{1,6}$, reported to be related to post-operative complications.

Of the seven patients (including the case reported herein) that received adjuvant radiotherapy after gross total resection, none had recurred over a short median follow-up period of eight months $s^{5,7,14,16,18}$. There was one reported death from sepsis at six months ${ }^{7}$.

Three patients received radiotherapy after a partial resection: one patient had tumor progression at 12 months, underwent two subsequent resections and chemotherapy ${ }^{19}$. The second patient died of tumor progression at 19 months $^{2}$. The third patient showed no evidence of tumor progression at two years followup $^{6}$. (Table 1)

\section{Radiotherapy}

The role of radiotherapy in the treatment of cerebellar liponeurocytoma remains unclear. Of the twelve patients who received radiotherapy after variable extents of resection ${ }^{2,5-}$ $7,9,14,16,18-20$, two have recurred within a median 1.8 year followup $^{2,19}$. On the other hand, the ten patients that did not receive radiotherapy had five documented recurrences over a median 6 year follow-up period ${ }^{5,6,8,12,21}$. Certainly, it can be argued that the higher recurrence rate in the subgroup not having received radiotherapy may be due to the longer follow-up rather than the effect of radiotherapy.

\section{Proliferation index}

Two previous cases report a proliferation index greater than $6 \%^{11,12,16,21}$. One patient had a recurrence 3.5 years after gross total resection ${ }^{11,12,21}$. The patient presented here remains without radiological evidence of recurrence at three years. Follow-up information was not provided in the third case $^{16}$. It appears reasonable to assume that tumors with a higher proliferation index will behave more aggressively and tend to recur earlier than those with a lower proliferation index. However, the available data (Table 2) is inconclusive in this regard given the small sample size and limited follow-up. Nonetheless, cerebellar liponeurocytomas have a relatively high recurrence rate, even after gross total resection, which becomes evident with longer follow-up ( $>8$ years). This may be especially so if the proliferation index is elevated. Since there are no universally accepted guidelines regarding the advisability and timing of radiotherapy in cases of cerebellar liponeurocytoma, we suggest that radiotherapy should be considered at least following incomplete resection and after complete resection of tumors with an elevated proliferation index. However, the potential morbidity of adjuvant radiotherapy must be kept in mind when tailoring treatment recommendations to individual patients based on limited clinical data.

Cerebellar liponeurocytoma is a newly recognized posterior fossa tumor with characteristic histological features. The proliferation index in this tumor is usually low and previous reports evoke a benign natural history. However, recurrence rates reach 50\% in reported cases of gross total resection without radiotherapy. Patients have a better prognosis following gross 
total resection. Patients with incomplete resection have a reserved prognosis and warrant consideration of adjuvant radiotherapy.

A higher proliferation index (greater than 6\%) may indicate a more aggressive course. Although supporting data is lacking, we suggest that adjuvant radiotherapy should be considered after complete resection of tumors with a high proliferation index.

\section{REFERENCES}

1. Bechtel JT, Patton JM, Takei Y. Mixed mesenchymal and neuroectodermal tumor of the cerebellum. Acta Neuropathol (Berl). 1978;15;41(3):261-3.

2. Aker FV, Ozkara S, Eren P, Peker O, Armagan S, Hakan T. Cerebellar liponeurocytoma/lipidized medulloblastoma. J Neurooncol. 2005;71(1):53-9.

3. Gonzalez-Campora R, Weller RO. Lipidized mature neuroectodermal tumour of the cerebellum with myoid differentiation. Neuropathol Appl Neurobiol. 1998;24(5):397-402.

4. Orlandi A, Marino B, Brunori M, Greco R, Spagnoli LG. Lipomatous medulloblastoma. Clin Neuropathol. 1997;16(4): 175-9.

5. Soylemezoglu F, Soffer D, Onol B, Schwechheimer K, Kleihues P. Lipomatous medulloblastoma in adults. A distinct clinicopathological entity. Am J Surg Pathol. 1996;20(4):413-8.

6. Giangaspero F, Cenacchi G, Roncaroli F, Rigobello L, Manetto V, Gambacorta M, Allegranza A. Medullocytoma (lipidized medulloblastoma). A cerebellar neoplasm of adults with favorable prognosis.[see comment]. Am J Surg Pathol. 1996; 20(6):656-64.

7. Davis DG, Wilson D, Schmitz M, Markesbery WR. Lipidized medulloblastoma in adults.[see comment]. Hum Pathol. 1993; 24(9):990-5.

8. Chimelli L, Hahn MD, Budka H. Lipomatous differentiation in a medulloblastoma. Acta Neuropathol (Berl)1991;81(4):471-3.

9. Horstmann S, Perry A, Reifenberger G, Giangaspero F, Huang H, Hara A, et al. Genetic and expression profiles of cerebellar liponeurocytomas. Brain Pathol. 2004;14(3):281-9.

10. Kleihues P, Louis DN, Scheithauer BW, Rorke LB, Reifenberger G, Burger PC, et al. The WHO classification of tumors of the nervous system. J Neuropathol Exp Neurol. 2002;61(3):215-25; discussion 26-9.

11. Buccoliero AM, Caldarella A, Bacci S, Gallina P, Taddei A, Di Lorenzo N, et al. Cerebellar liponeurocytoma: morphological, immunohistochemical, and ultrastructural study of a relapsed case. Neuropathology. 2005;25(1):77-83.

12. Cacciola F, Conti R, Taddei GL, Buccoliero AM, Di Lorenzo N. Cerebellar liponeurocytoma. Case report with considerations on prognosis and management. Acta Neurochir (Wien). 2002;144 (8):829-33

13. Kleihues P CL, Giangaspero F. Cerebellar Liponeurocytoma. 2nd ed. Kleihues P CW, editor. Lyon: IARC Press; 2000.

14. Owler BK, Makeham JM, Shingde M, Besser M. Cerebellar liponeurocytoma. J Clin Neurosci. 2005;12(3):326-9.

15. Alkadhi H, Keller M, Brandner S, Yonekawa Y, Kollias SS. Neuroimaging of cerebellar liponeurocytoma. Case report. J Neurosurg. 2001;95(2):324-31.

16. Alleyne $\mathrm{CH}$, Jr., Hunter S, Olson JJ, Barrow DL. Lipomatous glioneurocytoma of the posterior fossa with divergent differentiation: case report. Neurosurgery. 1998;42(3):639-43.

17. Ellison DW, Zygmunt SC, Weller RO. Neurocytoma/lipoma (neurolipocytoma) of the cerebellum. Neuropathol Appl Neurobiol. 1993;19(1):95-8.

18. Jackson TR, Regine WF, Wilson D, Davis DG. Cerebellar liponeurocytoma. Case report and review of the literature. J Neurosurg. 2001;95(4):700-3.

19. Jenkinson MD, Bosma JJ, Du Plessis D, Ohgaki H, Kleihues P, Warnke P, et al. Cerebellar liponeurocytoma with an unusually aggressive clinical course: case report. Neurosurgery. 2003;53(6):1425-7; discussion 8 .
20. Montagna N, Moreira D, Vaz LC, Reis M. Cerebellar liponeurocytoma: a newly recognized clinico-pathological entity. Arq Neuropsiquiatr. 2002;60(3-B):725-9.

21. Taddei GL, Buccoliero AM, Caldarella A, Conti R, Di Lorenzo N, Cacciola F, et al. Cerebellar liponeurocytoma: immunohistochemical and ultrastructural study of a case. Ultrastruct Pathol. 2001;25(1):59-63.

22. Kuchelmeister K, Nestler U, Siekmann R, Schachenmayr W. Liponeurocytoma of the left lateral ventricle--case report and review of the literature. Clin Neuropathol. 2006;25(2):86-94. 